\title{
A HIERARCHY FOR CIRCULAR CODES
}

\author{
Giuseppe Pirillo ${ }^{1,2}$
}

\begin{abstract}
We first prove an extremal property of the infinite Fibonacci* word $f$ : the family of the palindromic prefixes $\left\{h_{n} \mid n \geq 6\right\}$ of $f$ is not only a circular code but "almost" a comma-free one (see Prop. 12 in Sect. 4). We also extend to a more general situation the notion of a necklace introduced for the study of trinucleotides codes on the genetic alphabet, and we present a hierarchy relating two important classes of codes, the comma-free codes and the circular ones.
\end{abstract}

Mathematics Subject Classification. 68R15, 94A45.

À la Maison Heinrich Heine à l'occasion de son $50^{\mathrm{e}}$ anniversaire avec les remerciements de tout mon cœur pour le soutien qu'elle a donné pendant les 30 dernières années, à mes études et à mes recherches

Für das Heinrich-Heine-Haus aus Anlass seines 50-jährigen Bestehens mit meinem herzlichsten Dank für die Unterstützung meines Studiums und meiner wissenschaftlichen Arbeit in den letzten 30 Jahren

\section{INTRODUCTION}

The notion of a "code" has very different meanings in biology and theoretical computer science. In biology the "genetic code" associates the 64 trinucleotides with 20 amino acids. While in theoretical computer science a "code" is a set of uniquely decipherable words. Nevertheless, some notions in theoretical computer science, such as comma-free codes and circular codes, are useful in biology, see $[1,2,6]$. A theoretical method based on the notion of a necklace [20] has been

Keywords and phrases. Theory of codes, comma-free codes, circular codes.

1 IASI CNR, Viale Morgagni 67/A, 50134 Firenze, Italy; pirillo@math.unifi.it

2 Université de Marne-la-Vallée 5, boulevard Descartes Champs sur Marne 77454 Marne-la-

Vallée Cedex 2, France.

* Bigollo (Leonardo Pisano). 
useful for a complete description of the set of the trinucleotides self-complementary circular codes [21] and also for a detailed study of the trinucleotides comma-free codes [15].

We introduce the key notion of "tiling" (tessellation) of a word $w$ which is, roughly speaking, just a sequence $w_{1}, w_{2}, \ldots, w_{n}$ of words such that $w$ is a factor of $w_{1} w_{2} \cdots w_{n}$ (the sequence fills $w$ with no overlaps and no gaps in accordance with the Latin sense of the word "tessella", a small cubical piece used to make mosaics). When the $w_{i}$ belong to a finite set, even if there are potentially infinitely many tilings of a word $w$, only the minimal ones (see Defs. 1 and 2) are interesting. Using these notions of tilings and minimal tilings, we present here a general result, which "in nuce" was already in [20]: there is a hierarchy of codes $\mathcal{P}_{0}, \mathcal{P}_{1}, \ldots$, $\mathcal{P}_{n}, \ldots$ such that $\mathcal{P}_{0}$ is the class of comma-free codes and a finite code $X$ is circular if and only if there exists a positive integer $n$ such that $X$ is in class $\mathcal{P}_{n}$.

We like to conclude this introduction, pointing out that our result (which belongs to theoretical computer science) has pratically been suggested by the concrete study of the combinatorial properties of trinucleotides (which belongs to bio-informatics). More precisely, this result is a fruit of the reflection on the fact that, under certain conditions, the Arquès and Michel code (see [1,2]) allows us to retrieve the frame 0 using a window of length 13, i.e., four trinucleotide and a nucleotide.

\section{Preliminary Definitions And Properties}

We denote by $A$ an alphabet, by $A^{*}$ the free monoid on $A$, by $A^{+}$the free semigroup on $A$, by $\epsilon$ the empty word, and, finally, by $|u|$ the length of a word $u \in A^{*}$. We consider a word $u$ of length $k \geq 1$ as a map $u:\{1,2, \ldots, k-1, k\} \rightarrow A$; we write $u=u(1) u(2) \cdots u(i) \cdots u(k-1) u(k)$; we denote by $\widetilde{u}$ the mirror image $u(k) u(k-1) \cdots u(2) u(1)$ of $u$ and we say that a non-empty word $v$ is a palindrome if $v=\widetilde{v}$. A word $u$ is a factor of a word $v$ if there exist two words $u^{\prime}, u^{\prime \prime} \in A^{*}$ such that $v=u^{\prime} u u^{\prime \prime}$. When $u^{\prime}=\epsilon$ (resp. $\left.u^{\prime \prime}=\epsilon\right)$ we say that $u$ is a prefix (resp. suffix) of $v$. A proper factor (resp. proper prefix, proper suffix) $u$ of $v$ is a factor (resp. prefix, suffix) $u$ of $v$ such that $|u|<|v|$.

A (right) infinite word on $A$ is a map $q$ from the set of positive integers into $A$. We write $q=q(1) q(2) \cdots q(i) \cdots$. A word $u$ is a factor of $q$ if there exist a word $u^{\prime}$ and an infinite word $q^{\prime}$ such that $q=u^{\prime} u q^{\prime}$. If $u^{\prime}=\varepsilon$ we say that $u$ is a prefix of $q$. A non-empty word $u$ may be a factor of another (finite or infinite) word $w$ in more than one way. So it is useful to speak about occurrences. For this reason, let $i, j$ be integers such that $1 \leq i \leq j$ (with $j \leq|w|$ if $w$ is a finite word) and let us denote by $w(i, j)$ the factor $w(i) \cdots w(j)$ of $w$. We say that the pair of integers $(i, j)$ is an occurrence of the factor $u$ in the word $w$ if $u=w(i, j)$. We denote by $F(t)$ the set of all non-empty factors of a finite or infinite word $t$. Given a subset $X$ of $A^{*}$ we denote by $X^{n}$ the set of the words on $A$ which are product of $n$ words of $X$. We denote by $A^{\omega}$ the set of the infinite words on $A$ and by $X^{\omega}$ the set of the infinite words on $A$ which have the form $x_{1} x_{2} \cdots x_{i} \cdots$ with $x_{i} \in X$. 
Hereafter $\beta_{4}$ is the 4-letter genetic alphabet. A nucleotide is a letter of $\beta_{4}$. A domino (resp. trinucleotide) is a word of length 2 (resp. 3) over $\beta_{4}$. We denote by $\beta_{4}^{3}$ the set of all trinucleotides over $\beta_{4}$. For more details, see for example, $[1,2]$ or [20]. An ordered sequence $l_{1}, d_{1}, l_{2}, d_{2}, \ldots, d_{n-1}, l_{n}, d_{n}$ of nucleotides $l_{i}$ and dominoes $d_{i}$ is an $n$-necklace for a subset $X \subset \beta_{4}^{3}$ if $l_{1} d_{1}, l_{2} d_{2}, \ldots, l_{n} d_{n} \in X$ and $d_{1} l_{2}, d_{2} l_{3}, \ldots, d_{n-1} l_{n} \in X$. In [20], we proved that if $X$ is a subset of $\beta_{4}^{3}$ then the condition " $X$ is a circular code" is equivalent to the condition " $X$ has no 5-necklace".

Now imagine writing an infinite sequence (this is a concept extraneous to biology!) of trinucleotides $x_{i}$, with $x_{i} \in X$, where $X$ is a subset of $\beta_{4}^{3}$. Furthermore, imagine shifting the reading frame by one or two trinucleotides. Perhaps, we are able to read as a prefix just one trinucleotide of $X$ in the shifted sequence (in this case $X$ is not a comma-free code). Or maybe we are able to read as a prefix only the product of two, three, four, etc. consecutive trinucleotides of $X$. We might even be able to factorize the whole shifted sequence by trinucleotides of $X$. Anyway, if $X$ is a circular code, then we are able to read at most four consecutive trinucleotides of $X$. This corresponds to the window of Arquès and Michel, which has 13 nucleotides, and is also the meaning of the above result recalled from [20].

In [20], using the notion of a necklace, we characterized the (necessarily finite) languages of trinucleotides that are circular codes. In this paper, using the notion of a tiling, we characterize all the finite languages that are circular codes and we also present a hierarchy of circular codes.

Definition 1. Given an infinite word $s=s(1) s(2) \cdots s(i) \cdots$ and factors

$$
w=s(i, j), w_{1}=s\left(i_{1}, j_{1}\right), w_{2}=s\left(i_{2}, j_{2}\right), \ldots, w_{n}=s\left(i_{n}, j_{n}\right)
$$

of $s$, we say that $w_{1}, w_{2}, \ldots, w_{n}$ is a tiling (tessellation) of $w$ if $j_{1}+1=i_{2}$, $j_{2}+1=i_{3}, \ldots, j_{n-1}+1=i_{n}$ and $i_{1} \leq i \leq j \leq j_{n}$. We say that $w=s(i, j)$ is the trivial tiling of $w$.

Definition 2. We say that a tiling $s\left(i_{1}, j_{1}\right), s\left(i_{2}, j_{2}\right), \ldots, s\left(i_{n}, j_{n}\right)$ of $w=s(i, j)$ is minimal if $s\left(i_{2}, j_{2}\right), \ldots, s\left(i_{n-1}, j_{n-1}\right), s\left(i_{n}, j_{n}\right)$ is not a tiling of $w=s(i, j)$ and $s\left(i_{1}, j_{1}\right), s\left(i_{2}, j_{2}\right), \ldots, s\left(i_{n-1}, j_{n-1}\right)$ is not a tiling of $w=s(i, j)$. In other words, $s\left(i_{1}, j_{1}\right), s\left(i_{2}, j_{2}\right), \ldots, s\left(i_{n}, j_{n}\right)$ is minimal if $i_{1} \leq i<i_{2}$ and $j_{n-1}<j \leq j_{n}$.

Examples. Consider $f=f(1) f(2) \cdots f(i) \cdots=$ abaababaa $\cdots$ and note that $f(2,4)=b a a, f(5,7)=b a b$ is a minimal tiling of $f(3,6)=a a b a$. Note also that $a b a=f(4,6)$ is a minimal tiling of $a b a=f(4,6)$ (a factor is always a trivial tiling of itself and is clearly minimal) and, for each $n \leq 4$ and $n^{\prime} \geq 6$, the factor $f\left(n, n^{\prime}\right)$ is again a minimal tiling of $a b a=f(4,6)$. So minimal is intended in the sense that no unnecessary word is used on the left and on the right, i.e., at the beginning and end of the sequence of the occurrences. In Figure $1, x_{1}, x_{2}$ is a minimal tiling of $y_{1} ; x_{3}, x_{4}$ is a minimal tiling of $y_{2} ; x_{4}, x_{5}$ is a minimal tiling of $y_{3}$ but, for example, $x_{1}, x_{2} x_{3}$ is not a minimal tiling of $y_{1}$ and $x_{2}, x_{3} x_{4}$ is not a minimal tiling of $y_{2}$.

Definition 3. Let $s=s(1) s(2) \cdots s(i) \cdots$ be an infinite word and let $s(i, j)$ and $s\left(i^{\prime}, j^{\prime}\right)$ be two occurrences of the same factor $w$ of $s$. Then, given a tiling 


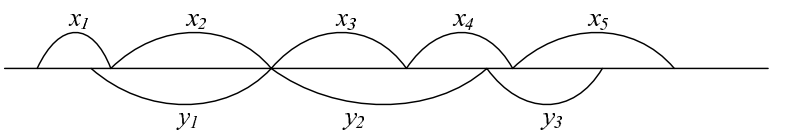

Figure 1. Examples of tilings.

$s\left(i_{1}, j_{1}\right), s\left(i_{2}, j_{2}\right), \ldots, s\left(i_{n}, j_{n}\right)$ of $s(i, j)$ and a tiling $s\left(i_{1}^{\prime}, j_{1}^{\prime}\right), s\left(i_{2}^{\prime}, j_{2}^{\prime}\right), \ldots, s\left(i_{n}^{\prime}, j_{n}^{\prime}\right)$ of $s\left(i^{\prime}, j^{\prime}\right)$, we say that these two tilings are equivalent if there exists factors $w_{1}$, $w_{2}, \ldots, w_{n}$ of $s$ such that $s\left(i_{1}, j_{1}\right)=s\left(i_{1}^{\prime}, j_{1}^{\prime}\right)=w_{1}, s\left(i_{2}, j_{2}\right)=s\left(i_{2}^{\prime}, j_{2}^{\prime}\right)=w_{2}, \ldots$, $s\left(i_{n}, j_{n}\right)=s\left(i_{n}^{\prime}, j_{n}^{\prime}\right)=w_{n}$ (see Fig. 2).

Definition 4. A subset $X$ of $A^{+}$is said to be a code over $A$ if for all $n, m \geq 1$ and $x_{1}, \ldots, x_{n}, x_{1}^{\prime}, \ldots, x_{m}^{\prime} \in X$ the condition

implies

$$
x_{1} \cdots x_{n}=x_{1}^{\prime} \cdots x_{m}^{\prime}
$$

$$
n=m \text { and } x_{i}=x_{i}^{\prime} \text { for } i=1, \ldots, n .
$$

Definition 5. Let $\left\{x_{\alpha}\right\}_{\alpha \geq 1}$ be a fixed infinite sequence of finite words from a set $X$ and let $x$ be an infinite sequence such that $x=x_{1} x_{2} \cdots x_{\alpha} \cdots \in X^{\omega}$. We say that the infinite set of integers $\left\{1=i_{1}, i_{2}, \ldots, i_{\alpha}, \ldots\right\}$ satisfying $i_{1}<$ $i_{2}<\cdots<i_{\alpha}<\cdots$ is the natural tiling set of $x$ if $x_{1}=x\left(i_{1}, i_{2}-1\right), x_{2}=$ $x\left(i_{2}, i_{3}-1\right), \ldots, x_{\alpha}=x\left(i_{\alpha}, i_{\alpha+1}-1\right), \ldots$ and we denote it by $T(x)$. If $y=$ $y_{1} y_{2} \cdots y_{n} \in X^{n}$ and $y_{1} y_{2} \cdots y_{n}=x\left(j_{1}, j_{2}-1\right) x\left(j_{2}, j_{3}-1\right) \cdots x\left(j_{n}, j_{n+1}-1\right)$ is an occurrence of $y$ in $x$, we say that the finite set of integers $\left\{j_{1}, j_{2}, \ldots, j_{n}, j_{n+1}\right\}$, with $j_{1}<j_{2}<\cdots<j_{n}<j_{n+1}$, is the local tiling set of this occurrence of $y$ in $x$ and, shortly, we denote it by $T(y)$.

The relationship between local tiling sets and natural tiling sets can be very different. Consider, for example, the first four occurrences of $a a$ in an infinite word $x$ in $X^{\omega}$ where $X$ is the suffix code $\{a, a a a b\}$ and $x$ begins with aaaaab.

Definition 6. Let $X$ be a subset of $A^{+}$and let $k$ be a non-negative integer. We say that $X$ has the property $\mathcal{P}_{k}$ if, for each infinite sequence $x \in X^{\omega}$ and local tiling set $T(y)$ of an occurrence of a factor $y$ in $x$, we have

$$
\operatorname{Card}(T(y) \backslash T(x)) \leq k .
$$

In other words, at most $k$ elements of $T(y)$ are not in $T(x)$. That is, the intersection of $T(y)$ with the complement of $T(x)$ has at most $k$ elements.

Examples. For each $k \geq 1$, the subsets $\left\{a^{k} b, a\right\}$ and $\left\{a b^{k}, b\right\}$ of $\{a, b\}^{+}$belong to $\mathcal{P}_{k}$, and the subset $\left\{a b^{k} c, b\right\}$ of $\{a, b\}^{+}$belongs to $\mathcal{P}_{k+1}$, as one can easily verify. Moreover $\{a, b\}$ and $\{a c, b\}$ belong to $\mathcal{P}_{0}$. In Figure 1 , an occurrence of $y=y_{1} y_{2} y_{3}$ in $x=x_{1} x_{2} x_{3} x_{4} x_{5} \cdots$ has a local tiling set with 3 integers which do not belong to the natural tiling set of $x$.

Proposition 1. If a subset $X$ of $A^{+}$has the property $\mathcal{P}_{k}$ for some non-negative integer $k$, then $X$ is a code.

Proof. Suppose, by way of contradiction, that $X$ has the property $\mathcal{P}_{k}$, for some integer $k$, but $X$ is not a code. Consider the equality $x_{1} x_{2} \cdots x_{n}=y_{1} y_{2} \cdots y_{m}$, 


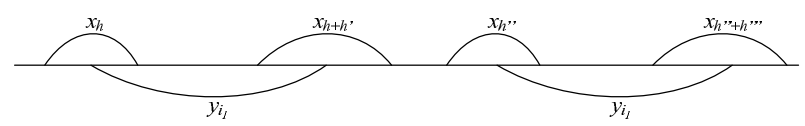

FigurE 2. The equivalent minimal tilings of $y_{i_{1}}$ and $y_{i_{2}}$.

where $x_{1} x_{2} \cdots x_{n}$ and $y_{1} y_{2} \cdots y_{m}$ are different, and suppose without loss of generality that $x_{1} \neq y_{1}$. Consider the infinite word $x=\left(x_{1} x_{2} \cdots x_{n}\right)^{\omega} \in X^{\omega}$. Then the word $y=y_{1} y_{2} \cdots y_{m}$ has an occurrence as a prefix of $x$ and $\operatorname{Card}(T(y) \cap(T(x)) \geq 1$. But clearly $y^{p}=\left(y_{1} y_{2} \cdots y_{m}\right)^{p}$ is again a prefix of $x$ and $\operatorname{Card}\left(T\left(y^{p}\right) \cap(T(x)) \geq p\right.$. Since this holds for any $p$, there is no positive integer $k$ such that $X$ has the property $\mathcal{P}_{k}$. Contradiction.

Definition 7. Let $X$ be a subset of $A^{+}$. We say that $X$ has the property $\mathcal{P}$ if $X$ has the property $\mathcal{P}_{k}$ for some non-negative integer $k$.

Proposition 2. If a subset $X$ of $A^{+}$has the property $\mathcal{P}$, then $X$ is a code.

Proof. It is very easy. Suppose that $X$ has the property $\mathcal{P}$. By definition, for some integer $k, X$ has the property $\mathcal{P}_{k}$, and hence $X$ is a code by Proposition 1 .

Definition 8. Given a code $X$ over $A$ we say that it is prefix (resp. suffix) if $u=v$ whenever $u, v \in X$ and $u$ is a prefix (resp. suffix) of $v$. A code is bifix if it is both a prefix code and a suffix code.

The set $X=\{a b, b a\}$ does not have the property $\mathcal{P}$, as one can easily see considering the factors $(b a)^{n}$ of $(a b)^{\omega}$, but it is a bifix code.

Definition 9. A code $X$ over $A$ is said to be comma-free if, for each $y \in X$ and $u, v \in A^{*}$ such that $u y v=x_{1} \cdots x_{n}$ with $x_{1}, \ldots, x_{n} \in X$, we have $u, v \in X^{*}$.

A comma-free code $X$ has the easiest deciphering [4]: if $w=x_{1} \cdots x_{n} \in X^{*}$ and $x \in F(w) \cap X$ then there is an integer $i, 1 \leq i \leq n$, such that $x=x_{i}$. We have

Proposition 3. A code $X$ has the property $\mathcal{P}_{0}$ if and only if it is comma-free.

Definition 10. A subset $X$ of $A^{+}$is a circular code over $A$ if, for each $n, m \geq 1$ and for each $x_{1}, \ldots, x_{n}, x_{1}^{\prime}, \ldots, x_{m}^{\prime}$ in $X, p \in A^{*}$ and $s \in A^{+}$, the conditions

$$
s x_{2} \cdots x_{n} p=x_{1}^{\prime} \cdots x_{m}^{\prime}
$$

and $x_{1}=p s$ imply $n=m, p=\varepsilon$ and, for $i=1, \ldots, n, x_{i}=x_{i}^{\prime}$.

\section{The infinite FibonacCi WORD}

Let $\varphi:\{a, b\}^{*} \rightarrow\{a, b\}^{*}$ be the morphism given by $\varphi(a)=a b, \varphi(b)=a$. The $n$-th finite Fibonacci word $f_{n}$ is defined in the following way: $f_{0}=b$ and, for each $n \geq 0, f_{n+1}=\varphi\left(f_{n}\right)$. For each $n \geq 2,\left|f_{n}\right|$ is the $n$-th element $F_{n}$ of the sequence of Fibonacci numbers. The infinite Fibonacci word $f$ (see, [7-9,11-14,16,18,19,22]) is the unique infinite word over $\{a, b\}$ such that, for each $n$, the word $f_{n}$ is a prefix of $f$. For each $n \geq 2$, we denote by $h_{n}$ the prefix of $f$ of length $\left|f_{n}\right|-2$. 
We will often consider some subsets of the family of palindromes $\left\{h_{n} \mid n \geq 3\right\}$ that are very useful examples of codes with the property $\mathcal{P}$.

Lemma 1 (below) is often useful in the study of properties of $f$. It belongs to the folklore and is very easy to prove. Item i) of the lemma is known as the "near-commutative property" (see [13]) and plays a central role in the combinatorics of the Fibonacci word. Note that, for each $n \geq 2, g_{n}=f_{n-2} f_{n-1}$ and a factor $v$ of $f$ is special if $v a, v b \in F(f)$.

Lemma 1. For each $n \geq 2$,

i) $f_{n}=h_{n} x y$ and $g_{n}=h_{n} y x$, where $x y=a b$ if $n$ is even and $x y=b a$ if $n$ is odd;

ii) $\left|h_{n}\right|=F_{n}-2$;

iii) $h_{n}$ is a special factor;

iv) $h_{n+3}=h_{n+1} x y h_{n} y x h_{n+1}$, where $x, y \in\{a, b\}, x \neq y$;

v) $h_{n}$ is a palindrome;

vi) $h_{n+2}=f_{n} h_{n+1}=h_{n+1} \tilde{f}_{n}=h_{n} \tilde{f}_{n+1}=f_{n+1} h_{n}$;

vii) for each integer $m \geq 0, h_{n}$ is a prefix and a suffix of $h_{n+m}$;

viii) $v \in F(f)$ if and only if $\widetilde{v} \in F(f)$.

Some properties of the family $\left\{h_{n} \mid n \geq 3\right\}$ also hold for the palindromic prefixes of a standard Sturmian word. See [5].

We will often use the following result of Karhumäki.

Proposition 4 [11]. The Fibonacci word $f$ is 4-power free, i.e., no factor of $f$ has the form $u^{4}$.

Let $u, v, w, z, z^{\prime} \in F(f)$. We say that $(u, v, w)$ is an overlap of $z$ and $z^{\prime}$ if $u v=z$, $v w=z^{\prime}$. When the central component $v$ of an overlap $(u, v, w)$ is non-empty, we say that the overlap is strict. In this case, $v$ is a proper suffix of $z$ and a proper prefix of $z^{\prime}$.

Proposition 5. Let $j \geq 3$. If $(u, v, w)$ is a strict overlap of $h_{j}$ and $h_{j}$ then we have $v \in\left\{h_{n} \mid n \geq 3\right\}$.

Proof. Let $v=v(1) v(2) \cdots v(k-1) v(k)$. For $1 \leq i \leq k$, we also have $v(i)=$ $h_{j}(i)=h_{j}\left(\left|h_{j}\right|-i+1\right)=v(k-i+1)$. So $v(i)=v(k-i+1)$ and $v$ is a palindrome. Since $v$ is a prefix of $h_{j}$ and consequently a prefix of $f$, we have $v \in\left\{h_{n} \mid n \geq 3\right\}$ by a well-known property of the palindromic prefixes of $f$ (the so called central words). See [5].

Remark 1. In [18] we studied the strict overlaps of the $f_{n}, g_{n}$ and $h_{n}$ under the supplementary condition that $u v w \in F(f)$ and we proved that, in this case, there are exactly two strict overlaps of $h_{n}$ and $h_{n}$, namely $\left(f_{n-1}, h_{n-2}, \widetilde{f}_{n-1}\right)$ and $\left(f_{n-2}, h_{n-1}, \widetilde{f}_{n-2}\right)$.

Several properties of the Fibonacci words belong, or are in relation, to the theory of codes. We presented in [19] some relations between Fibonacci numbers, Fibonacci words and the theory of codes. For example: if $f=u_{0} u_{1} \cdots u_{i} \cdots$ is the factorization of $f$ such that $\left|u_{i}\right|=F_{2 i+1}$ then $\left\{u_{i} \mid i \geq 0\right\}$ is a prefix code. We also proved a similar result with $\left|u_{i}\right|=F_{2(i+1)}$ and another one concerning a 
factorization of $f$ starting from the beginning and using only words of a bifix code. Related results are proved in $[10,17]$. Now, recall the following well-known

Proposition 6 [7]. Each element of the family $\left\{f_{n} \mid n \geq 1\right\}$ is a primitive word.

Proposition 7. Each element of the family $\left\{h_{n} \mid n \geq 3\right\}$ is a primitive word, with the only exception being abaaba $=h_{5}$.

Proof. Suppose, by way of contradiction, that $h_{j} \in\left\{h_{n} \mid n \geq 3\right\} \backslash\{a b a a b a\}$ is not primitive. Then $h_{j}=v^{k}$ with $k \geq 2$. If $k \geq 3$ then a cube is a prefix of $f$. But, by a preliminary result on fractional powers in the Fibonacci word [16], no cube is a prefix of $f$. So, there remains only the possibility $k=2$. In this case, using a result concerning squares in the Fibonacci word [22], we should have, for some $m>0, h_{j}=f_{m} f_{m}$. Observe that $h_{3}=a, h_{4}=a b a$, which clearly do not have the form $f_{m} f_{m}$ (while $h_{5}=a b a a b a=f_{3} f_{3}$ ). Now $\left|h_{6}\right|=11=F_{4}+F_{4}+F_{3}-2$, which is not even, and hence $h_{6}$ is not a square. In general, for $j \geq 7,\left|h_{j}\right|=F_{j}-2=$ $2 F_{j-2}+F_{j-3}-2$ from which it easily follows that, for $j \geq 7,\left|h_{j}\right|\left(i . e ., F_{j}-2\right)$ cannot be equal to $2 F_{m}$ for some $m$. So we reach a contradiction.

Corollary. Each singleton $\left\{h_{j}\right\}$, with $h_{j} \in\left\{h_{n} \mid n \geq 3\right\} \backslash\{a b a a b a\}$, is a commafree code.

Proof. Suppose, by way of contradiction, that the singleton $\left\{h_{j}\right\}$ with $j \geq 3, j \neq 5$, is not a comma-free code. Since $\left\{h_{j}\right\}$ is a singleton, there exists $u, v \in\{a, b\}^{*}$, such that $h_{j}^{\alpha}, \alpha \geq 2$, can be written as $h_{j}^{\alpha}=u h_{j} v$ where $u \neq h_{j}^{\beta}$ for any positive integer $\beta$. This implies that $h_{j} h_{j}$ contains an occurrence of $h_{j}$ that is different from those at the beginning and end. Hence, $h_{j}=w w^{\prime}=w^{\prime} w$ for some $w, w^{\prime} \in\{a, b\}^{+}$. Now, by a well-known result in the theory of words, we know that if two words commute, then they are powers of a common word (e.g., see [14]). Therefore, $w=v^{p}$ and $w^{\prime}=v^{q}$ for some word $v$ and positive integers $p, q$. But then $h_{j}=v^{p+q}$ where $p+q \geq 2$, contradicting the primitivity of $h_{j}$ (Prop. 7).

Lemma 2. Each element of the family $\left\{h_{n} \mid n \geq 3\right\} \backslash\{$ abaaba $\}$ is not the product of two or more elements of the family.

Proof. Suppose, by way of contradiction, that, for $j \neq 5$, the palindrome $h_{j}$ is the product of two or more elements of the family. This is not the case for $h_{3}=a$ and $h_{4}=a b a$. So $j \geq 6$. Suppose $h_{j}=u_{1} u_{2} \cdots u_{k}$ with $k \geq 2$ and $u_{i} \in\left\{h_{n} \mid n \geq\right.$ $3\} \backslash\{a b a a b a\}$ and note that $a$ is both a prefix and a suffix of each $u_{i}$. None of the $u_{i}$ can be equal to $a$. Otherwise, if $u_{1}=a$ then $h_{j}$ has $a a$ as a prefix, if $u_{k}=a$ then $h_{j}$ has $a a$ as a suffix and finally if $u_{i}=a, 1<i<k$, then $h_{j}$ has aaa as a factor, all of which are impossible.

So we have to prove that each element $h_{j}$ of the family $\left\{h_{n} \mid n \geq 3\right\} \backslash\{a b a a b a\}$ is not the product of two or more elements of the family $\left\{h_{n} \mid n \geq 4\right\} \backslash\{a b a a b a\}$.

Case $\mathbf{k}=2$. If $u_{1}=u_{2}=a b a$ we have $h_{j}=a b a a b a$. Contradiction. If $u_{1}=a b a$ and $u_{2} \neq a b a$ then $(a b a)^{3}$ is a prefix of $h_{j}$. Contradiction. If $u_{1} \neq a b a$ and 
$u_{2}=a b a$ then $(a b a)^{3}$ is a suffix of $h_{j}$. Contradiction. If $u_{1} \neq a b a$ or $u_{2} \neq a b a$ then $(a b a)^{4}$ is a factor of $h_{j}$. By Proposition 4 , we have a contradiction.

Case $\mathbf{k} \geq 3$. Consider $u_{1} u_{2} u_{3}$. If $u_{1}=u_{2}=u_{3}=a b a$ we have $h_{j}=(a b a)^{3}$. Contradiction. So at least one $u_{i}$ with $1 \leq i \leq 3$ is in $\left\{h_{n} \mid n \geq 6\right\}$. If this happens for exactly one $i, 1 \leq i \leq 3$, then $(a b a)^{3}$ is a prefix or a suffix of $h_{j}$. If this happens for at least two values of $i, 1 \leq i \leq 3$, then $(a b a)^{4}$ is a factor of $h_{j}$. Again, we have a contradiction, by Proposition 4.

It now remains to prove that each $h_{j}, j \neq 5$, is not the product of two or more elements of the family $\left\{h_{n} \mid n \geq 6\right\}$. In this case $u_{i} u_{i+1}$ (and consequently $h_{j}$ ) contains $(a b a)^{4}$ as a factor. So we have a contradiction by Proposition 4 .

The family $\left\{f_{n} \mid n \geq 1\right\}$ is not at all a code (indeed $f_{n+2}=f_{n+1} f_{n}$ ). But we do have:

Proposition 8. The family $\left\{h_{n} \mid n \geq 3\right\} \backslash\{a b a a b a\}$ is a code.

Proof. Suppose, by way of contradiction, that $\left\{h_{n} \mid n \geq 3\right\} \backslash\{a b a a b a\}$ is not a code. Consider the equality $x_{1} x_{2} \cdots x_{n}=y_{1} y_{2} \cdots y_{m}$ and, without loss of generality, suppose $x_{1} \neq y_{1}$. Suppose also, again without loss of generality, that $x_{1}$ is a prefix of $y_{1}$. Consider the smallest positive integer $i, 1 \leq i \leq n$, such that $y_{1}$ is a prefix of $x_{1} x_{2} \cdots x_{i}$. For some $u, v$ we have $x_{1} x_{2} \cdots x_{i}=x_{1} x_{2} \cdots x_{i-1} u v=y_{1} v$. So $y_{1}=x_{1} x_{2} \cdots x_{i-1} u$. The word $u$ is the central component of a strict overlap of $y_{1}$ and $x_{i}$ and, by Proposition 5, it belongs to $\left\{h_{n} \mid n \geq 3\right\}$. So $y_{1}$ is the product of at least two elements of $\left\{h_{n} \mid n \geq 3\right\}$ which contradicts Lemma 2.

Proposition 9. Each subset of $A^{+}$with at least two elements in the family $\left\{h_{n} \mid n \geq 3\right\} \backslash\{a b a a b a\}$ is not a comma-free code.

Proof. Suppose that $X$ is such that $\mid X \cap\left(\left\{h_{n} \mid n \geq 3\right\} \backslash\{\right.$ abaaba $\left.\}\right) \mid \geq 2$ and suppose that, for some positive integers $p, q, p<q$, the palindromes $h_{p}, h_{q}$ are in $X$. We claim that $\left\{h_{p}, h_{q}\right\}$ is not a comma-free code. Indeed, by Lemma 1 , for some $u \in\{a, b\}^{*}, h_{q}=h_{p} u$ and if, by way of contradiction, we suppose that $\left\{h_{p}, h_{q}\right\}$ is a comma-free code, then we also have $h_{q}=h_{p} u_{1} u_{2} \cdots u_{\alpha}$, for some $u_{1}$, $u_{2} \ldots, u_{\alpha} \in\left\{h_{p}, h_{q}\right\}^{\alpha}, \alpha \geq 1$. That is, $h_{q}$ is the product of two or more elements of $\left\{h_{n} \mid n \geq 3\right\}$, which contradicts Lemma 2. Now, $X$ cannot be a comma-free code because it contains a subset which is not a comma-free code.

Proposition 10. The family $\left\{h_{n} \mid n \geq 3\right\} \backslash\{a b a a b a\}$ is a circular code.

Proof. Suppose, by way of contradiction, that $\left\{h_{n} \mid n \geq 3\right\} \backslash\{a b a a b a\}$ is not a circular code. Then, there exist $x_{1}, \ldots, x_{n}, x_{1}^{\prime}, \ldots, x_{m}^{\prime}$ in $\left\{h_{n} \mid n \geq 3\right\} \backslash\{$ abaaba $\}$, $p \in\{a, b\}^{+}$and $s \in\{a, b\}^{+}$, such that $s x_{2} \cdots x_{n} p=x_{1}^{\prime} \cdots x_{m}^{\prime}$ and $x_{1}=p s$. Note that $s$ and $p$ are central components of strict overlaps of $x_{1}$ and $x_{1}^{\prime}$ and of $x_{m}^{\prime}$ and $x_{1}$ respectively. So, by Proposition 5, s, $p \in\left\{h_{k} \mid k \geq 3\right\}$. Since $x_{1}$ is the product of two elements of $\left\{h_{k} \mid k \geq 3\right\}$ and since $x_{1} \neq a b a a b a$, we reach a contradiction by Lemma 2 or by Proposition 8 . 


\section{A HIERARCHY}

In this section, we give a characterization of all the finite languages that are circular codes (Th. 1). A first relationship between our property $\mathcal{P}$ and some classical notions of theory of codes (limited codes and uniformly synchronous codes) is in Theorem 2. Finally, the classes $\mathcal{P}_{0}, \mathcal{P}_{1}, \ldots, \mathcal{P}_{k}, \mathcal{P}_{k+1}, \ldots$ constitute a hierarchy of codes (Prop. 11).

Theorem 1. Given an alphabet $A$ and a finite subset $X$ of $A^{+}$, the following conditions are equivalent:

i) $X$ has the property $\mathcal{P}$;

ii) $X$ is a circular code.

Proof. i) implies ii). Suppose that $X$ has the property $\mathcal{P}$. By Proposition 2, X is a code. Suppose, by way of contradiction, that $X$ is not a circular code. Then there exist $x_{1}, \ldots, x_{n}, x_{1}^{\prime}, \ldots, x_{m}^{\prime} \in X, p \in A^{+}$and $s \in A^{+}$, such that $s x_{2} \cdots x_{n} p=$ $x_{1}^{\prime} \cdots x_{m}^{\prime}, x_{1}=p s$ and the conditions of Definition 10 are not satisfied. Consider the infinite word $x$ :

and its natural tiling set

$$
x_{1} x_{2} \cdots x_{n} x_{1} x_{2} \cdots x_{n} \cdots x_{1} x_{2} \cdots x_{n} \cdots=\left(x_{1} x_{2} \cdots x_{n}\right)^{\omega}
$$

$$
j_{1}=1<j_{2}<\cdots<j_{n}<\cdots
$$

Note that $x$ has a factor $u$ which admits two factorizations

$$
u=s x_{2} \cdots x_{n} p=x_{1}^{\prime} \cdots x_{m}^{\prime} .
$$

Consider an occurrence of $x_{1}^{\prime} \cdots x_{m}^{\prime}$ in $x$ and its local tiling set:

$$
j_{1}^{\prime}<j_{2}^{\prime}<\cdots<j_{m}^{\prime}<j_{m+1}^{\prime}
$$

For each integer $\alpha \geq 1$, the element $j_{\alpha}^{\prime}$ does not belong to $\left\{j_{1}, j_{2}, \ldots, j_{n}, \ldots\right\}$, otherwise, since $\mathrm{X}$ is a code, we reach a contradiction.

So $x_{1}^{\prime} \cdots x_{m}^{\prime}$ has an occurrence with $m+1$ elements of its local tiling set in the complement of the natural tiling set of $x=\left(x_{1} x_{2} \cdots x_{n}\right)^{\omega}$.

In a similar way, for each positive integer $\beta$, the factor $\left(x_{1}^{\prime} \cdots x_{m}^{\prime}\right)^{\beta}$ has an occurrence with $\beta m+1$ elements of its local tiling set in the complement of the natural tiling set of $x=\left(x_{1} x_{2} \cdots x_{n}\right)^{\omega}$.

Thus for each positive integer $k, X$ does not have the property $\mathcal{P}_{k}$ and consequently does not have the property $\mathcal{P}$. Contradiction.

Proof. ii) implies i). Suppose that $X$ is a circular code and, by way of contradiction, suppose that $X$ does not have the property $\mathcal{P}$, i.e., there is no positive integer $k$ such that $X$ has the property $\mathcal{P}_{k}$.

For each positive integer $k$, there exist $x=x_{1} x_{2} \cdots x_{\alpha} \cdots \in X^{\omega}$ (having $\left\{i_{1}=\right.$ $\left.1, i_{2}, \ldots, i_{\alpha}, \ldots\right\}$ as a natural tiling set), a factor $y$ of $x$ such that $y=y_{1} y_{2} \cdots y_{n}$ (with $y_{i} \in X$ ) and the local tiling set $\left\{j_{1}, j_{2}, \ldots, j_{n}, j_{n+1}\right\}$ of an occurrence of $y$ in $x$ has more than $k$ elements in the complement of $\left\{i_{1}=1, i_{2}, \ldots, i_{\alpha}, \ldots\right\}$. This means that at least $k$ factors $y_{i}$ of $y$ have a minimal non-trivial tiling set with the $x_{i}$. 
Since $X$ is finite and each factor of $x$ has finitely many minimal non-trivial tiling sets with the $x_{i}$, we can choose $k$ (for example $k=(2 M+3) \delta \cdot|X|+1$, where $\delta$ is the maximum number of minimal non-trivial tiling sets of an element $z \in X$ and $M$ is the maximum of the lengths of elements of $X$ ) such that two $y_{i}$, say $y_{i_{1}}$ and $y_{i_{2}}$, satisfy $y_{i_{1}}=y_{i_{2}}=w$ for some $w$. Furthermore, for suitable integers $h, h^{\prime}, h^{\prime \prime}$, $h^{\prime \prime \prime}, h^{\prime \prime}>h+h^{\prime}$, the minimal tiling of $y_{i_{1}}$ is $x_{h}, \ldots, x_{h+h^{\prime}}$, the minimal tiling of $y_{i_{2}}$ is $x_{h^{\prime \prime}}, \ldots, x_{h^{\prime \prime}+h^{\prime \prime \prime}}$ and $x_{h}=x_{h^{\prime \prime}}, \ldots, x_{h+h^{\prime}}=x_{h^{\prime \prime}+h^{\prime \prime \prime}}$. In other words, the minimal tilings of $y_{i_{1}}$ and $y_{i_{2}}$ are equivalent. See Figure 2. Moreover, there exist words $p, s$ and $p^{\prime}, s^{\prime}, p \neq \epsilon, p^{\prime} \neq \epsilon$, such that the tilings of the occurrences $y_{i_{1}}$ and $y_{i_{2}}$ of $w$ satisfy

Now, we have

$$
\begin{gathered}
x_{h}=p s=x_{h^{\prime \prime}}, x_{h+h^{\prime}}=p^{\prime} s^{\prime}=x_{h^{\prime \prime}+h^{\prime \prime \prime}}, \\
p y_{i_{1}} s^{\prime}=x_{h} \cdots x_{h+h^{\prime}}, \quad y_{i_{1}}=s x_{h+1} \cdots x_{h+h^{\prime}-1} p^{\prime}, \\
p y_{i_{2}} s^{\prime}=x_{h^{\prime \prime}} \cdots x_{h^{\prime \prime}+h^{\prime \prime \prime}} \\
s^{\prime} x_{h+h^{\prime}+1} \cdots x_{h^{\prime \prime}-1} p=y_{i_{1}+1} \cdots y_{i_{2}-1} .
\end{gathered}
$$

$$
\begin{gathered}
s x_{h+1} \cdots x_{h+h^{\prime}-1}\left(x_{h+h^{\prime}}\right) x_{h+h^{\prime}+1} \cdots x_{h^{\prime \prime}-1} p \\
=s x_{h+1} \cdots x_{h+h^{\prime}-1}\left(p^{\prime} s^{\prime}\right) x_{h+h^{\prime}+1} \cdots x_{h^{\prime \prime}-1} p \\
=\left(s x_{h+1} \cdots x_{h+h^{\prime}-1} p^{\prime}\right)\left(s^{\prime} x_{h+h^{\prime}+1} \cdots x_{h^{\prime \prime}-1} p\right) \\
=y_{i_{1}} y_{i_{1}+1} \cdots y_{i_{2}-1} .
\end{gathered}
$$

Since, by assumption, $p$ is non-empty, $X$ is not a circular code. Contradiction.

We refer to [4] for the definitions of limited codes and uniformly synchronous codes. Combining our Theorem 1 with Theorem 2.6 of [4] we have

Theorem 2. Given an alphabet $A$ and a finite subset $X$ of $A^{+}$, the following conditions are equivalent:

i) $X$ has the property $\mathcal{P}$;

ii) $X$ is a circular code;

iii) $X$ is a limited code;

iv) $X$ is a uniformly synchronous code.

The hierarchy is justified by the following very easy

Proposition 11. A code $X$ in $A^{+}$which has the property $\mathcal{P}_{k}$ also has the property $\mathcal{P}_{k+1}$.

Remark 2. In the first two classes of our hierarchy there are codes whose elements are factors of the Fibonacci word. Indeed $\{a b, b\}$ and $\{a, a b\}$ belong to the class $\mathcal{P}_{1}$. Moreover, by Lemma 1 , the family $\left\{h_{k} \mid k \geq 6\right\}$ is very far from being bifix, so it cannot be comma-free (see [4]). Anyway, we will see now (Prop. 12) that $\left\{h_{k} \mid k \geq 6\right\}$ belongs to the class $\mathcal{P}_{2}$ and, in this sense, it is "almost" a comma-free code. Note that the relation $\left\{h_{k} \mid k \geq 6\right\} \in \mathcal{P}_{2}$ requires only a short proof, presented hereafter. On the other hand, some relations (for example $\left.X=\left\{h_{n} \mid n \geq 3\right\} \backslash\{a b a a b a\} \in \mathcal{P}_{4}\right)$ require arguments which, as far as we know at the moment, are too long to be contained in this paper. In a forthcoming paper we will discuss these relations.

The following result shows that the family $\left\{h_{k} \mid k \geq 6\right\}$ is "almost" comma-free. 
Proposition 12. The code $\left\{h_{k} \mid k \geq 6\right\}$ belongs to the class $\mathcal{P}_{2}$.

Proof. Set $X=\left\{h_{k} \mid k \geq 6\right\}$ and consider an infinite sequence $x=x_{1} \cdots x_{i} \cdots$ $\in X^{\omega}$, its natural tiling set $\left\{i_{1}=1, i_{2}, \ldots, i_{\alpha}, \ldots\right\}$ and a factor $y$ of $x$ with $y=$ $y_{1} y_{2} \cdots y_{n}, y_{i} \in X$. Suppose, by way of contradiction, that the local tiling set $\left\{j_{1}, j_{2}, \ldots, j_{n}, j_{n+1}\right\}$ of an occurrence of $y$ in $x$ has more than 3 elements in the complement of the natural tiling set of $x$. Without loss of generality we can suppose that exactly three integers $i, j, k, i<j<k$, belong to this local tiling set. Consider $\beta \in\{1, \ldots, n-1\}$ such that $y_{\beta}=x\left(j^{\prime}, j-1\right)$ and $y_{\beta+1}=x\left(j, j^{\prime \prime}\right)$ for some $j^{\prime}, j^{\prime \prime}$. Set $y_{\beta}=v$ and $y_{\beta+1}=w$. Consider also, in the natural tiling set of $x$, the greatest integer $i_{\gamma}$ that is smaller than $j$. Then, in the natural tiling set of $x$, the smallest integer that is greater than $j$ is $i_{\gamma+1}$. We have four possibilities:

Case 1. $i_{\gamma} \leq j^{\prime}$ and $i_{\gamma+1} \geq j^{\prime \prime}$. In this case $x\left(i_{\gamma}, i_{\gamma+1}-1\right)$ contains $(a b a)^{4}$. By Proposition 4 , we have a contradiction.

Case 2. $i_{\gamma}>j^{\prime}$ and $i_{\gamma+1} \geq j^{\prime \prime}$. In this case $x\left(i_{\gamma}, j-1\right)$ is the central component of a strict overlap of two elements of $X$ and, by Proposition 5 , it belongs to $\left\{h_{k} \mid k>\right.$ $3\}$. If $x\left(i_{\gamma}, j-1\right)=a$ then $w$ must begin with $b$. Contradiction. If $x\left(i_{\gamma}, j-1\right)=a b a$ then $w$ must begin with ababa. Contradiction. If $x\left(i_{\gamma}, j-1\right)=a b a a b a$ then $w$ must begin with $b$. Contradiction. Finally, if $x\left(i_{\gamma}, j-1\right) \in\left\{h_{k} \mid k>3\right\}$ then $x\left(i_{\gamma}, i_{\gamma+1}\right)$ must contain $(a b a)^{4}$, which is again a contradiction by Proposition 4 .

Case 3. $i_{\gamma} \leq j^{\prime}$ and $i_{\gamma+1}<j^{\prime \prime}$. In this case $x\left(j, i_{\gamma+1}-1\right)$ is the central component of a strict overlap of two elements of $X$ and, by Proposition 5, it belongs to $\left\{h_{k} \mid k>3\right\}$. If $x\left(j, i_{\gamma+1}-1\right)=a$ then $v$ must end with $b$. Contradiction. If $x\left(j, i_{\gamma+1}-1\right)=a b a$ then $v$ must end with ababa. Contradiction. If $x\left(j, i_{\gamma+1}-\right.$ $1)=a b a a b a$ then $v$ must end with $b$. Contradiction. Finally, if $x\left(j, i_{\gamma+1}-1\right) \in$ $\left\{h_{k} \mid k>3\right\}$ then $x\left(i_{\gamma}, i_{\gamma+1}\right)$ must contain $(a b a)^{4}$, which is again a contradiction by Proposition 4 .

Case 4. $i_{\gamma}>j^{\prime}$ and $i_{\gamma+1}<j^{\prime \prime}$. In this case $x\left(i_{\gamma}, j-1\right)$ is the central component of a strict overlap of two elements of $X$ and, by Proposition 5, it belongs to $\left\{h_{k} \mid k>3\right\}$. With the the same arguments as above, $x\left(j, i_{\gamma+1}-1\right)$ belongs to $\left\{h_{k} \mid k>3\right\}$. Consequently $x\left(i_{\gamma}, i_{\gamma+1}-1\right)$ is the product of two elements of $\left\{h_{k} \mid k>3\right\}$. By Lemma 2 we have a contradiction.

The result of Proposition 12 is optimal. Indeed $h_{7}=a b a a b a b a a b a a b a b a a b a=$ $a b a a b a b a h_{6}=h_{6} a b a b a a b a$ and $h_{7} h_{7}=a b a a b a b a h_{6} h_{6} a b a b a a b a$. So $h_{7}^{\omega}$ has an occurrence of $h_{6} h_{6}$ with local tiling set $9,20,30$. Since 9 and 30 are in the complement of $1,20,39, \ldots, 1+19 n, \ldots$ we have $\left\{h_{k} \mid k \geq 6\right\} \notin \mathcal{P}_{1}$.

Acknowledgements. We thank Dipartimento di Matematica "U. Dini" for giving us a friendly hospitality, Jacques Justin for very helpful conversations, the two referees for their interesting remarks and Amy Glen for a very accurate revision of the manuscript. 


\section{REFERENCES}

[1] D.G. Arquès and C.J. Michel, A complementary circular code in the protein coding genes. J. Theor. Biol. 182 (1996) 45-58.

[2] D.G. Arquès and C.J. Michel, A circular code in the protein coding genes of mitochondria. J. Theor. Biol. 189 (1997) 273-290.

[3] J. Berstel, Mots de Fibonacci. Séminaire d'informatique théorique. LITP, Paris (1980-81) $57-78$.

[4] J. Berstel and D. Perrin, Theory of codes. Academic Press (1985).

[5] J. Berstel and P. Seebold, Sturmian words, in Algebraic Combinatorics on words, edited by M. Lothaire. Cambridge University Press (2002).

[6] F.H.C. Crick, J.S. Griffith and L.E. Orgel, Codes without commas. Proc. Natl. Acad. Sci. USA 43 (1957) 416-421.

[7] A. de Luca, A combinatorial property of the Fibonacci words. Inform. Process. Lett. 12 (1981) 193-195.

[8] A. de Luca, Sturmian words: structure, combinatorics, and their arithmetics. Theoret. Comput. Sci. 183 (1997) 45-82.

[9] X. Droubay, Palindromes in the Fibonacci word. Inform. Process Lett. 55 (1995) 217-221.

[10] J. Justin and G. Pirillo, On some factorizations of infinite words by elements of codes. Inform. Process. Lett. 62 (1997) 289-294.

[11] J. Karhumäki, On cube-free $\omega$-words generated by binary morphism. Discrete Appl. Math. 5 (1983) 279-297.

[12] D.E. Knuth, The Art of Computer Programming. Addison-Wesley, Reading, Mass. (1968).

[13] D.E. Knuth, J.H. Morris and V.R. Pratt, Fast pattern matching in strings. SIAM J. Comput. 6 (1977) 323-350.

[14] M. Lothaire, Combinatorics on words. Addison-Wesley (1983).

[15] C.J. Michel, G. Pirillo and M.A. Pirillo, Varieties of comma-free codes. Comput. Math. Appl. (in press).

[16] F. Mignosi and G. Pirillo, Repetitions in the Fibonacci infinite word. RAIRO-Theor. Inf. Appl. 26 (1992) 199-204.

[17] G. Pirillo, Infinite words and biprefix codes. Inform. Process Lett. 50 293-295 (1994).

[18] G. Pirillo, Fibonacci numbers and words. Discrete Math. 173 (1997) 197-207.

[19] G. Pirillo, Some factorizations of the Fibonacci word. Algebra Colloquium 6 (1999) 361-368.

[20] G. Pirillo, A characterization for a set of trinucleotides to be a circular code, In Determinism, Holism, and Complexity, edited by C. Pellegrini, P. Cerrai, P. Freguglia, V. Benci and G. Israel. Kluwer (2003).

[21] G. Pirillo and M.A. Pirillo, Growth function of self-complementary circular codes. Biology Forum 98 (2005) 97-110.

[22] P.P. Séébold, Propriétés combinatoires des mots infinis engendrés par certains morphismes. PhD. thesis, L.I.T.P., Paris. (1985).

Received January 26, 2007. Accepted November 22, 2007. 